\title{
LA INVESTIGACIÓN HISTÓRICA Y EL DEBATE ACTUAL SOBRE LA SALUD MASCULINA: EL CASO DE ALEMANIA
}

\author{
Martin Dinges \\ Institut für Geschichte der Medizin \\ der Robert Bosch Stiftung \\ martin.dinges@igm-bosch.de \\ www.igm-bosch.de
}

Recibido: 26 junio 2013; Aceptado: 4 octubre 2013.

Cómo citar este artículo/Citation: Dinges, Martin (2014), "La investigación histórica y el debate actual sobre la salud masculina: el caso de Alemania", Asclepio 66 (1): p040. doi: http://dx.doi.org/10.3989/asclepio.2014.14

RESUMEN: El discurso actual sobre la salud masculina remite con demasiada insistencia a una imagen obsoleta y monolítica de la masculinidad contrastando de manera simplista el colectivo "hombres" con el de las "mujeres". Sin embargo, el análisis histórico hace muy evidentes los notables cambios que se han producido en la salud de los hombres, tanto en lo que se refiere a sus actitudes como a sus comportamientos, desde la revolución industrial; así, por ejemplo, se ha transformado no solamente la vida profesional y el mundo del trabajo, sino también la relación entre la masculinidad y el comportamiento de riesgo a lo largo de la vida y, asimismo, la evaluación médica de ciertos comportamientos de alto riesgo como fumar. Las diferencias en la esperanza de vida entre hombres y mujeres merecen una explicación más precisa y menos simplista. Desde una perspectiva histórica de longue-durée, también es un mito que los hombres guarden silencio en asuntos de salud.

PALABRAS CLAVE: salud masculina; masculinidad; comportamientos de salud; esperanza de vida; mundo del trabajo; comportamientos de riesgo; uso de los servicios médicos; hábito de fumar tabaco; Alemania; siglos XIX, XX y XXI.

\section{HISTORICAL RESEARCH AND THE CURRENT DEBATE ABOUT MEN'S HEALTH: THE GERMAN CASE}

\begin{abstract}
The current discourse on men's health refers too insistently to an obsolete and monolithic pattern of masculinity contrasting "men" with "women" in a simplistic manner. However, historical analysis shows the remarkable changes that have occurred in the health of men, both in terms of their attitudes and their behaviors, since the industrial revolution; so, for example, the relationship between masculinity and risk behavior throughout life, the professional life and the world of work, and also the medical evaluation of certain high-risk behaviors such as smoking. Differences of the life expectancy between men and women deserve an explanation more accurate and less simplistic. From a historical perspective of longue-durée, it is also a myth that men keep silent on matters of health.
\end{abstract}

KEY WORDS: Men's health; masculinity; health behavior; life expectancy; labor market; risk behavior; use of medical services; smoking; Germany; XIX, XX and XXI centuries.

Copyright: () 2014 CSIC. Este es un artículo de acceso abierto distribuido bajo los términos de la licencia Creative Commons Attribution-Non Commercial (by-nc) Spain 3.0. 


\section{INTRODUCCIÓN}

A la luz de los estudios epidemiológicos y antropológicos, la salud de los hombres ofrece a menudo una imagen negativa y, desde luego, siempre problemática. Su esperanza de vida es muy inferior a la de las mujeres y la forma en que cuidan de su salud deja mucho que desear: son totalmente sedentarios o hacen ejercicio en exceso, tienen malos hábitos alimenticios, fuman mucho y beben demasiado alcohol (sobre todo ante las adversidades). Además, no se toman en serio los síntomas corporales ni hablan sobre sus problemas de salud; no expresan sus dolencias y con frecuencia se vuelven agresivos contra los demás o contra sí mismos. Consultan con sus médicos cuando ya es demasiado tarde, o no consultan con ellos en absoluto. Se suelen saltar las revisiones médicas y apenas cumplen los tratamientos que se les prescriben.

Este «discurso sobre la salud masculina» no es más que una pequeña muestra de la gran cantidad de material publicado en las revistas en particular y en la literatura científica en general. Con razón el sociólogo Michael Meuser lo denomina un «discurso del déficit», ya que pone de relieve la debilidad, si no la inferioridad, de los hombres en el ámbito de la salud y el bienestar (Meuser, 2007, pp. 73-86). En ocasiones, agrega Meuser, da la impresión incluso de que sea necesario sermonear a los hombres: si no cuidan más de su salud, se les castigará con la enfermedad o con una corta vida.

La cuestión es que, pese a que la información que tenemos sobre la salud masculina está estadísticamente bien documentada y a que los expertos tratan de promover la concienciación acerca del problema, los discursos del déficit casi nunca llegan a las personas a las que les conciernen más directamente. Ante un mensaje que critica el comportamiento deficiente o inadecuado de un sujeto, éste suele reaccionar mediante el rechazo y la negación.

Llegados a este punto, me gustaría destacar un enfoque que se practica en educación para la salud, según el cual se pueden obtener mejores resultados con refuerzos positivos. Este enfoque se emplea en las jornadas de información sobre la salud masculina organizadas por profesionales de la salud. En mi opinión, el discurso sobre la salud masculina debería abstenerse de hacer comparaciones de forma general y alejarse de la imagen negativa y parcial que se tiene de los hombres. En lugar de tratar de alarmarles con pronósticos pesimistas, deberíamos centrarnos en aquellos aspectos de su comportamiento relativos a la salud que ofrecen una buena base para una discusión más matizada y polifacética acerca de las conexiones entre la masculinidad y la salud. A esto puede contribuir la investigación histórica.
Primero: El actual discurso sobre la salud masculina hace referencia con demasiada frecuencia a una imagen obsoleta y monolítica de la masculinidad.

Hollstein describe la «masculinidad tradicional» vigente durante los últimos veinte años como un complejo de predisposiciones conductuales expansivas, agresivas, insensibles, posesivas y sedientas de poder; en definitiva, tales comportamientos son malos para la salud. Su idea es sencillamente que la «masculinidad tradicional» perjudica la salud (Hollstein, 2002, pp. 53-66). De esto también podría deducirse que los hombres son unos auténticos «idiotas de la salud» (Dinges, 2009, pp. 19-23). Esta crítica, como la mayoría de las estadísticas divulgadas, es el resultado de una comparación que contrapone dos grandes poblaciones: los hombres y las mujeres. Este enfoque oculta, al menos en el discurso público, las considerables diferencias existentes dentro del grupo de los hombres. Aunque puede que la mayoría de los hombres cuide poco de su salud, en la investigación epidemiológica se ha identificado una parte de la población masculina que se preocupa mucho por su bienestar. La media estadística, que representa el comportamiento de la mayoría, no basta para dar por hecho afirmaciones generales sobre una "esencia de la masculinidad». Tener en cuenta sólo a la mayoría implica que se omite a la minoría de dos maneras: en relación a los problemas de salud y en relación a la construcción del comportamiento masculino general. Por lo tanto, los discursos de este tipo no tienen en cuenta ni los comportamientos saludables de los hombres ni el denominado comportamiento masculino «no tradicional», de acuerdo con la deliberada simplificación de Hollstein. Este discurso tan contrapuesto impide reconocer comportamientos masculinos más saludables que pueden apreciarse a lo largo de la historia y que, a su vez, podrían constituir un recurso para promover el cambio (Hoffmann, 2010, pp. 130 y 137; Schweig, 2008, pp. 118 y 156; Dinges, 2011, p. 33; Dinges, 2012, p. 130).

Segundo: La investigación médica tiende a favorecer el pensamiento simplista que compara de manera reducida el colectivo "hombres" con el de las «mujeres».

Para facilitar la investigación tiene sentido dividir los colectivos de pacientes en mujeres y hombres. Esta separación de acuerdo con el sexo es un requisito cada vez más importante en, por ejemplo, pruebas de medicamentos, estudios de medicina basada en la evidencia y estudios sobre los servicios sanitarios. Esto se debe a que las medicinas, los tratamientos y la estructura de los servicios sanitarios afectan de diferente forma a hombres y a mujeres. Este tipo de investigaciones que diferencia entre ambos sexos todavía se consideran deseables en muchas áreas de es- 
tudio. Además, en la Unión Europea es una exigencia política, ya que la transversalidad de la perspectiva de género (gender mainstreaming), viene siendo un requisito imprescindible desde hace algunos años, y su aplicación es ineludible. Las buenas políticas se apoyan en las conclusiones de investigaciones relevantes como base para la toma de decisiones. La división convencional entre hombres y mujeres conlleva el peligro de promover la idea de dos mundos separados, al codificar conceptos de género opuestos (Babitsch, 2005). Así pues, los estudios que apuntan a la igualdad de género no deben contribuir a la fijación de estereotipos en los que el sexo biológico sea un criterio fundamental, cosa en la que es relativamente fácil caer. Esto puede conducir a nuevas ideas ficticias sobre comportamientos que se presuponen esencialmente masculinos.

Tercero: La salud masculina se ve fuertemente influida por el desarrollo histórico.

Como apuntaré seguidamente con algunos ejemplos, este aserto hace referencia a la esperanza de vida al nacer, el mundo laboral y la vida profesional, la actitud de los hombres hacia la salud, la conexión entre comportamientos de riesgo y masculinidad, y el uso que hacen los hombres de la asistencia médica y los medicamentos.

En general, los cambios acontecidos son considerables. En su mayoría, han surgido a lo largo de un período de tiempo relativamente corto, hablando en términos históricos, y algunos han tenido lugar muy recientemente, en las últimas generaciones. En otras palabras, el estado de salud de los hombres y su conducta en relación a la salud y a la enfermedad son el resultado de procesos históricos. Por lo tanto, aunque vuelvan a cambiar o a sufrir modificaciones, no son en absoluto la expresión esencial de la masculinidad. En lugar de atribuir el comportamiento de los hombres a una supuesta masculinidad arquetípica, la perspectiva histórica permite una percepción más clara de los cambios que se han ido sucediendo en el pasado.

Cuarto: El desarrollo histórico se hace más evidente al estudiar los considerables cambios que ha sufrido la diferencia de esperanza de vida entre hombres y mujeres a lo largo del tiempo.

El mejor indicativo del estado de salud de una población es la esperanza de vida media al nacer. Este dato también desempeña una función primordial en el debate actual sobre la salud masculina. Hoy en día, en la mayoría de las sociedades posindustriales, las mujeres viven mucho más tiempo que los hombres. Concretamente en Alemania viven en torno a cinco años y medio más. ¿Cómo se explica esta diferencia? Los factores a tener en cuenta, así como su relevancia, son objeto de debate científico y médico. Un estudio realizado con decenas de miles de frailes y monjas que vivían en monasterios y conventos sugiere que las mujeres tienen una ventaja biológica de esperanza de vida de alrededor de un año. Esto nos deja cuatro años y medio de diferencia por explicar. Se podría deducir del estudio de los frailes y las monjas que una vida en la que hay seguridad económica y social, con poca competitividad y relaciones sociales asiduas y estables, parece ser muy saludable. Además, el uso reducido de las «libertades nocivas» como fumar y beber, también contribuye a una mayor esperanza de vida de los frailes. Por lo pronto, este dato apunta a un par de razones que podrían explicar la existencia de esa diferencia de género.

Desde el punto de vista del historiador son de especial interés los importantes cambios de la brecha de género en cuanto a la esperanza de vida (Weigl, 2007, pp. 23-40). En Alemania, en 1850, ambos sexos tenían una esperanza de vida al nacer cercana a los 40 años. Después se redujo para los hombres en casi cuatro años, y entre las mujeres en sólo uno, lo que significa que en los años 1880/1890 la diferencia se había quedado en torno a los tres años a favor de las mujeres (36 y 39 años, respectivamente). En 1900/1910, ambos sexos tenían una mayor esperanza de vida $(44,8$ y 48,3$)$, y las mujeres vivían tres años y medio más. En aquella época, mejoraron las condiciones de higiene en los partos. Para el año 1950, la brecha se había ampliado ligeramente, a cuatro años de diferencia, con una mayor esperanza de vida de las mujeres. Después, durante la época de la reconstrucción de Alemania, mejoró con más rapidez: en 1960 la diferencia de esperanza de vida entre hombres y mujeres era de 5,5 años y, a partir de 1970, se elevó a 6,5 años. Continuó creciendo lentamente hasta 1985, llegando a 6,7 años, y en 2002 la ventaja de las mujeres se redujo a 5,7 años, siendo actualmente de 5 años (Weiland, 2006, pp. C874-C879). La tendencia existente desde 1850 de una mayor esperanza de vida de las mujeres respecto a la de los hombres está revirtiendo desde mediados de la década de 1980. Esto podría deberse al esfuerzo por conseguir la igualdad en las condiciones de vida de hombres y mujeres.

Quinto: Este desarrollo histórico se debe a diversos factores.

Desde el punto de vista de la historia de la salud, la industrialización benefició menos a los hombres que a las mujeres en lo relativo a la esperanza de vida. El ritmo de los cambios muestra que durante los períodos de la primera y la segunda Revolución Industrial, así como el cuarto de siglo que siguió a la reconstrucción de Alemania tras 1945, los hombres se vieron mucho más afectados que las mujeres.

De acuerdo con esto podemos afirmar que durante la segunda Revolución Industrial el riesgo profesional 
Tabla 1. Esperanza de vida al nacer de hombres y mujeres en Alemania (1850-2010) ${ }^{1}$

\begin{tabular}{lccc}
\hline Año & Hombres & Mujeres & $\begin{array}{c}\text { Ventaja en la esperanza de vida de las mujeres respecto a los } \\
\text { hombres }\end{array}$ \\
\hline 1850 & 40 & 40 & 0 no hay diferencia \\
\hline $1880 / 1890$ & 36 & 39 & 3 años más \\
\hline $1900 / 1910$ & 44,8 & 48,3 & $\mathbf{3 , 5}$ años más \\
\hline $1949 / 51$ & 64,6 & 68,5 & 4,1 años más \\
\hline $1960 / 62$ & 66,9 & 72,4 & 5,5 años más \\
\hline $1970 / 72$ & 67,4 & 73,8 & 6,4 años más \\
\hline $1980 / 82$ & 70,2 & 76,9 & 6,7 años más \\
\hline $1990 / 92$ & 72,9 & 79,3 & 6,4 años más \\
\hline $2009 / 11$ & 77,7 & 82,7 & 5 años más \\
\hline
\end{tabular}

específica de cada género, a menudo inevitable, fue un factor perjudicial para la salud mucho más influyente para los hombres que su supuesta predisposición "típicamente masculina» de exponerse voluntariamente a los peligros. Los trabajadores poco cualificados que migraban de zonas rurales tenían entonces tan poca libertad de decisión como la tienen en la actualidad. El concepto de «masculinidad tradicional» encubre los importantes cambios a lo largo de la historia, así como las enormes desigualdades entre, por ejemplo, los hombres que reciben una mejor educación y los que no. Asimismo, dentro del conjunto de exposiciones perjudiciales para la salud, algunos factores individuales son más significativos en unos períodos históricos que en otros. Los sistemas políticos también influyeron en esto, y no sólo durante la época nazi. Para ambos sexos, la esperanza de vida en la República Democrática Alemana era inferior a la de la República Federal Alemana. Sin embargo, en los primeros siete años tras la reunificación, la esperanza de vida en la República Democrática Alemana se ajustó casi totalmente a los niveles de Alemania Occidental, para las mujeres algo más rápidamente que para los hombres. Esta diferencia de ritmo sugiere que, más allá de la mera "masculinidad tradicional», hubo una serie de factores que fueron responsables de estos cambios. Esto se confirma cuando analizamos las subidas y bajadas en la esperanza de vida, ya que están relacionadas con edades totalmente diferentes. Antes de la I Guerra Mundial, la mortalidad infantil se redujo mucho y de manera definitiva. Después de la guerra, la mortalidad de la gente joven disminuyó. Finalmente, después de la II Guerra Mundial la población empezó a vivir cada vez más años, hasta llegar en grandes cohortes a edades avanzadas. Como las epidemias han desaparecido en Alemania casi por completo, en la actualidad la mortalidad se debe principalmente a enfermedades cardiovasculares y degenerativas.

Sexto: La vida profesional y el mundo del trabajo han cambiado considerablemente los recursos y los riesgos para la salud a lo largo de las cinco últimas generaciones.

Desde la década de 1980, se aprecia una diferencia decreciente en la esperanza de vida de hombres y mujeres, lo cual sugiere que debemos analizar más de cerca los cambios en las condiciones laborales. Este análisis puede hacerse considerando períodos de unos 33 años por generación, empezando con la construcción del primer ferrocarril en Alemania (1835). Durante la primera generación, el trabajo siguió siendo predominantemente rural, aunque ya había comenzado la migración a las ciudades y las condiciones laborales de la era industrial. La actividad aumentó tremendamente durante la Segunda Industrialización, y una gran cantidad de mano de 
obra masculina se incorporó a puestos de trabajo que eran perjudiciales para la salud. Además, debido al desarraigo por la migración, sus condiciones de vida se hicieron precarias. El trabajo de las mujeres siguió desarrollándose principalmente en el hogar, lo que implicaba una mejor nutrición y mejores condiciones de vida en general. Tan sólo un pequeño número de mujeres trabajaron en la industria y también se vieron expuestas a considerables riesgos para la salud. La mayoría de las mujeres trabajadoras solían acabar casándose y dejando sus empleos en las fábricas tras unos ocho años de trabajo por término medio, y raramente volvían a trabajar. Para los hombres la vida laboral consistía en un trabajo fuera del hogar a tiempo completo, y para toda la vida. Entretanto, para las mujeres este tipo de empleos normalmente se limitaba, en ocasiones en contra de su voluntad, a la época previa al matrimonio, debido a convenciones sociales o incluso a prohibiciones laborales. La proporción de mujeres empleadas se mantuvo extremadamente estable a largo plazo. Pese a pequeñas fluctuaciones a corto plazo, ascendía a exactamente el $36 \%$ en los años de las muestras analizadas (1882; $1907 ; 1925 ; 1939 ; 1950$ y 1972 ).

El trabajo que solían desempeñar los hombres presentaba mayores riesgos para la salud ${ }^{2}$. En términos generales, la separación imperante entre el desempeño de trabajos más peligrosos por hombres y el desempeño de trabajos sedentarios, de escritura y de tipo administrativo, por hombres y mujeres, se extiende hasta el segundo tercio del siglo XX. Después, la dicotomía se agrava aún más y el riesgo de mortalidad masculina aumenta debido al servicio militar obligatorio durante las dos guerras mundiales, que a menudo duraba varios años. Los riesgos para la salud de los hombres a largo plazo también eran ligeramente superiores debido a la guerra, y a que eran tomados como prisioneros. Tras el período de reconstrucción que sucedió a la II Guerra Mundial, la última generación del siglo XX redujo relativamente el trabajo físico duro debido a los progresos técnicos y al traslado de los procesos de producción básicos a otros países, lo que condujo a la correspondiente reducción de los riesgos para la salud en Alemania.

Pese a los rápidos cambios que suceden en torno a 1970, las mujeres siguieron trabajando a tiempo parcial en mayor medida que los hombres. Casi el 94\% de los hombres empleados en 2004 tenían un trabajo a tiempo completo, frente a sólo el 57,9\% de las mujeres. El $85,4 \%$ de todos los contratos a tiempo parcial los realizaban mujeres. Esta proporción sigue en aumento ${ }^{3}$. Además, el $10 \%$ de los hombres hacía horas extras, frente al $5 \%$ de las mujeres. Las horas extras realizadas durante cierto tiempo contribuyen a aumentar el riesgo de enfermedades cardiovasculares (Siegrist, 2010, p. 79).
Habría que distinguir importantes diferencias dentro de este esbozo general de los cambios, teniendo en cuenta las diferentes regiones y etapas de la vida. No obstante, demuestra que el trabajo y la vida profesional cambiaron de manera significativa, pero conservaron unas importantes constantes generales propias de cada género.

Los riesgos para la salud derivados de puestos de trabajo peligrosos siempre son más aplicables a los hombres durante la totalidad de su vida laboral. El hecho de que el porcentaje de mujeres que desempeñaban trabajos de oficina creciera rápidamente desde la época de la República de Weimar no presentó ninguna desventaja sobre la salud de las mujeres (Weigl, 2011, p. 132).

No obstante, el trabajo también puede ser un recurso para la salud (Hoffmann, 2007, pp. 241-256). En algunos relatos personales, los hombres mencionan a sus compañeros de trabajo como importantes asesores en cuestiones de salud. Posiblemente este tipo de redes sea menos relevante para las mujeres porque ellas tienden a contar con redes sociales más amplias. En cualquier caso, fue a partir de 1850 cuando el trabajo cobró mayor importancia para su comportamiento, el de los hombres, en relación a la salud, coincidiendo con un creciente alejamiento del hogar. Sin embargo, no sabemos en detalle cómo afectó a la salud de los hombres, dado que el desempleo también implicaba un riesgo para la salud en aquellos hombres que dependían de un trabajo a tiempo completo. El paradigma masculino asociado al trabajo industrializado a jornada completa dificulta el cambio hacia una actitud más saludable. Pese a todo, la jornada completa sigue siendo el indicador principal en la vida profesional de los hombres. Se considera que el empleo a tiempo parcial que desempeñan casi exclusivamente las mujeres es más saludable porque proporciona mayor autonomía y satisfacción personal. No debe sorprender que las madres con un trabajo a tiempo parcial estén mucho más sanas que las que sólo son amas de casa (Resch, 2002, p. 405). Muchos problemas de salud de los hombres tienen que ver con su vida laboral, resultado de la «modernización» del trabajo durante los últimos 150 años.

Séptimo: Existe, a lo largo de la vida, una relación variable entre la masculinidad y el comportamiento de riesgo que también afecta a la salud de los hombres de diversas maneras.

La actitud de los hombres hacia la salud varía considerablemente en cada etapa de su vida. Entre la pubertad y la formación de una pareja para casarse, se observan con mayor frecuencia comportamientos perjudiciales para la salud que en la edad adulta. Por otra parte, para algunos hombres los comportamientos de riesgo pueden continuar for- 
mando parte de su vida adulta. El entusiasmo de los hombres jóvenes por los deportes de riesgo es tan típico en esta etapa de la vida como la conducción peligrosa tras el consumo de alcohol.

Históricamente, este comportamiento de riesgo (post)adolescente da lugar a consecuencias muy diversas. Los accidentes de coche mortales empezaron a tener devastadoras tasas de mortalidad entre los hombres jóvenes con el inicio de la motorización masiva, en la década de 1960. La reducción en muertes masculinas en la carretera desde 1980 no se debe tanto a un cambio en la «masculinidad tradicional» sino a las vallas protectoras y a la mejora de los servicios de emergencia. Esto ha elevado la esperanza de vida de los hombres en casi medio año.

Por otro lado, los «juegos peligrosos y competitivos de la masculinidad" son, en gran medida, previsibles y socialmente aceptados. A los hombres jóvenes se les sigue elogiando si muestran bravura en situaciones de peligro y cuando resisten al dolor, porque esta actitud les hace aptos para muchos trabajos y tareas sociales como, por ejemplo, el ejército. Puede que esta fuera la verdadera razón por la que se criaba a los niños para que se hicieran "duros», especialmente entre los años 1880 y 1960.

No obstante, muchos hombres relatan que trataron de protegerse de peligros en los puestos de trabajo industriales o durante la guerra, teniendo mucho cuidado o evitando tomar riesgos (Bourke, 1996). Por lo tanto, aparte del comportamiento adquirido con el que se les crió y de que se les impusiera la obligación de ser arriesgados, los hombres a menudo también mostraron las tendencias opuestas. Los discursos de salud que se basan en evitar riesgos tienen que lidiar con la proyección social de una imagen determinada de la masculinidad, sobre todo durante la adolescencia (Dinges, 2011a). Sin embargo, el desarrollo histórico en el mundo laboral que hemos descrito demuestra que cada vez se exige menos de los hombres esa temeraria disposición de tomar riesgos. Paradójicamente, esto podría motivar a los hombres jóvenes a buscar desafíos y a obtener el reconocimiento simbólico, de una «masculinidad tradicional» que empieza a perder su relevancia de hecho, en el culturismo, el sexo y el alcohol.

Los discursos médicos que pretendan evitar los riesgos criticando los comportamientos perjudiciales para la salud de los jóvenes deberían tener en cuenta las razones históricas que hay detrás de esa educación encaminada hacia la dureza y la reciedumbre, así como los recientes cambios en el mundo del trabajo.
Octavo: Los marcadores de género y la evaluación médica de ciertos comportamientos de alto riesgo han cambiado considerablemente a lo largo de la historia.

En la imagen de la «masculinidad tradicional» y en el actual discurso de la salud masculina, la idea de hombría se asocia estrechamente a comportamientos que son perjudiciales para la salud. Sin embargo, el ejemplo del tabaco revela que la práctica de fumar y su significado han cambiado mucho a lo largo de la historia.

En el siglo XIX fumar se consideraba una práctica restringida casi exclusivamente a los hombres, con la excepción de algunas mujeres, como las prostitutas y las artistas. A partir del año 1900, aproximadamente, las mujeres empezaron a ser el blanco de los anuncios de cigarrillos. Se hacían continuas alusiones a la emancipación, que acabaron personificándose socialmente en la figura de la «nueva mujer» de la República de Weimar. Sólo a partir de la década de 1960 las mujeres fumadoras fueron aceptadas en público casi sin restricciones, así como las mujeres que tomaban bebidas alcohólicas fuertes. Este cambio fue sucediendo a lo largo de varias generaciones, y parece ser el motivo por el que fumar todavía se considera un signo de la emancipación de las mujeres en los espacios públicos. Esta idea la comparten tanto historiadoras feministas como la industria del tabaco. El concepto de emancipación podría explicar todavía por qué fuman tantas chicas jóvenes ${ }^{4}$.

Históricamente, la masculinidad y el tabaco siempre han estado relacionados. Para muchos chicos el primer cigarrillo simboliza la entrada en la vida adulta; es casi como un rito de iniciación. En consecuencia, fumaban, y aún a día de hoy fuman, muchos más hombres que mujeres, lo que contribuye a una mayor mortalidad masculina por el consumo de tabaco. Sin embargo, en el discurso público el vínculo entre el rol de género masculino y el comportamiento perjudicial para la salud no está tan claro.

Es anacrónico el argumento de que, antes de comenzar a fumar, los hombres "siempre» se habían comportado de modo que perjudicaban su salud. Puede que esto sea objetivamente cierto en el año 2013, pero subjetivamente tanto los hombres como las mujeres no solían estar bien informados sobre los efectos del tabaco. En Alemania, la crítica de que fumar es cancerígeno y malo para la salud apareció y llegó a un público general en la década de 1920 (Dinges, 2012, p. 136). El gobierno nazi realizó una serie de campañas antitabaco. Aunque la profesión médica pudo haber advertido sobre los riesgos de fumar a lo largo de las décadas siguientes, hasta los años sesenta se subestimaron los peligros del tabaco. Con la ayuda de los anuncios de la industria tabacalera, el tabaco gozó de una imagen positiva en la sociedad y fue ampliamente aceptado hasta los años noventa. Teniendo en cuenta estos mensajes contradictorios, el tabaco no puede considerarse un "comportamiento de riesgo típicamente masculino", 
ya que el riesgo no se hizo evidente hasta hace poco. Anteriormente, la gente era mucho menos consciente de los peligros del tabaco que en la actualidad ${ }^{5}$.

Noveno: Es un mito que los hombres guarden silencio en asuntos de salud.

La moderna investigación científica participa de la idea de que los hombres no hablan lo suficiente sobre sus problemas de salud y que, cuando lo hacen, ya es demasiado tarde, y que no consultan con un experto médico competente cuando debieran hacerlo. Se dice que en relación a sus parejas, o bien se quejan sobre la más mínima dolencia o bien se guardan para sí los problemas más graves.

Sin embargo, la investigación histórica halla gran cantidad de evidencia en cartas, diarios y autobiografías de que a los hombres les gustaba expresar sus pensamientos sobre asuntos relacionados con la salud y la enfermedad en muchas y variadas situaciones. Aparte de hablar de sus dolencias en pareja, en el siglo XIX las reuniones sociales mixtas eran la ocasión preferida para sacar a la luz los problemas de salud, como puede verse en informes y cartas de la época (Dinges, 2002, pp. 25f.). Asimismo, la correspondencia entre parejas casadas y prometidas a menudo contiene referencias a cuestiones de salud. Las parejas incluso desarrollan roles de género, aconsejándose mutuamente sobre estos temas. Aunque es más frecuente que las mujeres adopten este rol en una relación, los hombres también son bastante activos como consultores de salud, en calidad de parejas, padres y hermanos (Schweig, 2007, pp. 211-226). Algo similar sucede con los amigos.

Las autobiografías escritas por hombres también contienen referencias a asuntos de salud, tanto en la escrita por el sencillo mercenario de la Guerra de los Treinta Años como en la más popular autobiografía del siglo XX. Si se analizan más de cerca estas fuentes se descubre que los hombres de todas las clases sociales sabían de la existencia de los recursos de salud y hacían uso de ellos. Por sólo aludir a unos cuantos ejemplos de los siglos XIX y XX, ya fueran trabajadores o emprendedores, prisioneros políticos o combatientes, todos presentaban comportamientos muy similares para promover y proteger la salud, pese a que los medios económicos disponibles estaban distribuidos de forma desigual.

Estos documentos históricos sitúan las estadísticas actuales en otra perspectiva. Ya no se puede decir sin más que la actitud de los hombres hacia su salud sea descuidada en general y esté basada en una relación de negligencia hacia sus cuerpos. Tiene mucho más sentido emplear la evidencia histórica como base para la investigación en contextos y situaciones en las que los hombres sí se han preocupado por su salud.
Décimo: Los hombres solían hacer un uso distinto de los servicios médicos.

El hecho de que los hombres se muestren reacios a hacer uso de los servicios médicos en oferta se asocia críticamente con la «masculinidad tradicional», que prohíbe buscar ayuda a los hombres. Hasta 1995 el $60 \%$ de todos los pacientes en las consultas médicas en Alemania eran mujeres. Aún teniendo en cuenta la proporción ligeramente mayor de mujeres en el total de la población, la diferencia de uso sigue siendo del $16,8 \%$. Si a esto le restamos las actuales «necesidades ginecológicas», consecuencia de la medicalización de los últimos 150 años, seguimos teniendo una diferencia del $12,2 \%$ para la que no hay explicación.

La tasa del $60 \%$ de mujeres frente al $40 \%$ de hombres no demuestra antropológicamente que los hombres tengan menos voluntad de buscar ayuda, porque la presencia de los hombres en las consultas de los médicos ha variado a lo largo de los últimos siglos. Entre 1800 y $1850-1860$ a veces había más mujeres y a veces había más hombres. Sin embargo, antes de 1800 , predominaban los pacientes masculinos en las consultas médicas. El hecho decisivo que hizo que la mayoría de los pacientes fuesen mujeres tuvo lugar a mediados del siglo XIX. Desde entonces, la cifra del $60 \%$ (en 2008/11: 58\%) se ha mantenido asombrosamente constante pese a los avances habidos en el campo de la medicina ${ }^{6}$. Estos cambios, acontecidos en la primera mitad del siglo XIX, tuvieron en parte que ver con la supuesta mejoría de los servicios médicos relacionados con el parto. Esto condujo a una mayor afluencia de mujeres a las consultas de los médicos; consultas que, a su vez, solían acabar derivando a la paciente a otros especialistas. Las mujeres aprendieron así un nuevo habitus de salud según el cual acudían a los especialistas en mayor medida que los hombres (Dinges, 2007a, pp. 293-320).

Lo significativo de todo esto es la transformación que tuvo lugar. No se puede interpretar el cambio histórico en el sentido de que los hombres buscaban ayuda médica cada vez menos o sólo en algunas ocasiones. Más bien eran las mujeres quienes hacían un uso cada vez mayor de la asistencia médica desde la década de 1860 . Esto se debía, en principio, a sus particulares necesidades de salud y a los servicios que se les ofrecía. El que esto tuviera o no una influencia positiva en su comportamiento general en relación a la salud es otro asunto. El desarrollo histórico es el resultado de una relación más reciente entre las mujeres y los médicos que ha ido en aumento a lo largo de la historia. Esta relación tiene tan poco que ver con la «feminidad esencial» como la relación entre el comportamiento masculino y una "masculinidad» atemporal. De esto se deduce el siguiente aserto. 
Undécimo: La toma de medicamentos por parte de mujeres es el resultado de un proceso histórico y no puede convertirse en un modelo para los hombres.

Es un hecho ampliamente conocido que las mujeres toman más medicinas que los hombres. En la literatura encontramos dudas respecto a si este consumo es, o no, aconsejable desde el punto de vista médico. La toma de medicamentos se ve fuertemente determinado por los médicos, sobre todo en el caso de medicamentos psicotrópicos disponibles sólo bajo receta. Por lo tanto, se puede afirmar que la toma de medicamentos por parte de las mujeres es un ejemplo más de su medicalización, que consultan con mayor frecuencia a los médicos, quienes, a su vez, tienden a diagnosticarles en mayor medida desórdenes mentales.

Esto queda confirmado por las conclusiones de estudios históricos. La reciente investigación sobre el consumo de medicamentos según el género muestra que, en los siglos XVIII y XIX, en Alemania y los EE.UU. los hombres consumían más medicamentos que las mujeres. Durante el siglo XVIII, en Núremberg el consumo de los hombres triplicaba al de las mujeres (Blessing, 2011, p. 86). De nuevo, el punto de inflexión es la década de 1850. Desde ese momento el consumo de medicamentos por parte de las mujeres es cada vez mayor (Hoffmann, 2013). De esto se deduce que las actitudes propias de cada género hacia el consumo de medicamentos no están relacionadas con una «masculinidad» o «feminidad» inherente o esencial. Son el resultado, por un lado, de los discursos médicos y antropológicos y, por otro, de la oferta médica, que han ido moldeando a lo largo de la historia los comportamientos de hombres y mujeres.

Este ejemplo genera una reflexión crítica acerca del «discurso del déficit» del comportamiento de los hombres hacia su salud. Puede que los hombres recurran menos a tomar medicamentos porque son menos sensibles al dolor. Con respecto al consumo de medicamentos, esto no es necesariamente una actitud negativa? ${ }^{7}$. Más problemático es el discurso parcial sobre la salud que siempre etiqueta el comportamiento de las mujeres como positivo. En este caso, por ejemplo, se trataría de una «percepción más diferenciada de los síntomas».
En cualquier caso, puede que la evidencia médica sugiera que hacer un uso más frecuente de los servicios sanitarios no es necesariamente lo mejor para la salud de los hombres. Esto quiere decir que la manera de recurrir a la medicina por parte de los hombres debería verse de un modo diferenciado, más que de un modo exclusivamente negativo.

Duodécimo: La historia de la salud masculina proporciona una visión más distanciada, y por lo tanto más amplia y contrastada, acerca de la salud de los hombres, que la que adoptan las ciencias de la salud, que se centran principalmente en el presente inmediato.

La investigación en salud estudia los comportamientos actuales en relación a la salud y los problemas asociados a estas conductas. Con frecuencia tienen una fuerte vertiente de género, como por ejemplo respecto al uso de los servicios médicos, bien sea con fines diagnósticos o terapéuticos. El comportamiento de los pacientes, los diagnósticos, la investigación, los discursos sobre la medicalización y los servicios médicos son factores que pueden contribuir a la creación de estereotipos de género que se asumen precipitadamente como si fuesen naturales o "genuinamente» masculinos.

Debido a la distancia respecto a la situación presente, los estudios históricos permiten una mejor comprensión de las constelaciones de género, que solían ser muy distintas en el pasado. En lugar de insistir, como hace Hollstein, en una «masculinidad esencial» que supuestamente conduce a comportamientos perjudiciales, el enfoque histórico abre de manera heurística perspectivas mucho más interesantes, como por ejemplo la distinción entre generaciones de hombres y entre diferentes edades de la vida (Dinges, 2013, 2011a). El estudio histórico defiende, junto con las ciencias de la salud, una diferenciación dentro de la comunidad "masculina» que tenga en cuenta las situaciones económicas y sociales y las diferentes etapas de la vida, y que se emplee el género como una categoría relacional. Siempre deberíamos tratar de dar una explicación al comportamiento de los hombres o de las mujeres dentro de un contexto concreto, como puede ser un mercado médico determinado. Finalmente, una perspectiva histórica nos permite comprender mejor los condicionantes y las consecuencias de la medicalización, que no deberían subestimarse. 


\section{NOTAS}

1 Statistisches Bundesamt, Periodensterbetafeln für Deutschland. Allgemeine und abgekürzte Sterbetafeln 1871/1881 bis 2004/2006, publicado el 28. 3. 2008, Wiesbaden 2008, 401414 para todos datos desde 1870. Datos para 1850 según Imhof, Arthur E. (1990), Lebenserwartungen in Deutschland vom 17. bis 19. Jahrhundert, Weinheim, VCH acta humaniora, $462 \mathrm{f}$. (Perioden); para 2009/2011. Véase: https://www.destatis.de/ DE/ZahlenFakten/GesellschaftStaat/Bevoelkerung/Sterbefaelle/Tabellen/LebenserwartungDeutschland.html [consultado el 12/7/2013].

2 Para una reflexión acerca del reconocimiento más tardío de las enfermedades profesionales en las mujeres, véase: Lengwiler, 2007, pp. 257-274.

3 En el mes de marzo de 2004, de un total de 7,17 millones de contratos a tiempo parcial, 6,13 millones correspondían a mujeres. En ese mismo año, $93,8 \%$ de los hombres (97,1 \% en 1991) trabajaban a jornada completa, mientras que solo lo hacían un $57,9 \%$ (69,8 \% en 1991) de las mujeres. Statistisches Bundesamt, Leben und Arbeiten in Deutschland, Ergebnisse des Mikrozensus 2004, Tabellenanhang, Tab. Nr. 21. Teilzeitbeschäftigung von Frauen: 1991: 1,8 Mio; 2004: 6,1 Mio (Marzo 2004; p. 44)

El informe de 2004 demuestra también que los hombres hacen el doble de horas extras que las mujeres: $10 \%$ en lugar de $5 \% ; p$. 47 Statistisches Bundesamt, Datenreport 2006: 42,3\% ratio de

\section{BIBLIOGRAFÍA}

Babitsch, Birgit (2005), Soziale Ungleichheit, Geschlecht und Gesundheit. Bern, Huber.

Blessing, Bettina (2011), "Geschlechtsspezifische Arzneimitteltherapien im 18. Jahrhundert", Österreichische Zeitschrift für $\mathrm{Ge}$ schichtswissenschaften, 22 (2), pp. 75-93.

Bourke, Joanna (1996), Dismembering the male: men's bodies, Britain and the Great War. London, Reaktion Books.

Dinges, Martin (2002), "Männergesundheit in Deutschland: Historische Aspekte". En: Jacobi, Günther (ed.), Praxis der Männergesundheit, Stuttgart, Thieme, pp. 24-33.

Dinges, Martin (ed.) (2007), Männlichkeit und Gesundheit im historischen Wandel ca. 1800 - ca. 2000. Stuttgart, Steiner.

Dinges, Martin (2007a), "Immer schon 60\% Frauen in den Arztpraxen? Zur geschlechtsspezifischen Inanspruchnahme des medizinischen Angebotes (1600-2000)". En Dinges, 2007, pp. 293-320.

Dinges, Martin (2009), "Männer, die beratungsresistenten Gesundheitsidioten?", Blickpunkt Der Mann. Wissenschaftliches Journal für Männergesundheit, 7 (1), pp. 19-23. mujeres en todas personas con empleo; $52,2 \%$ de las mujeres casadas tienen empleo (datos para 2004). Esta tendencia de la feminización del empleo a tiempo parcial continúa en el mismo sentido, como lo demuestra el reciente informe: Keller, Matthias; Haustein, Thomas (2012), „Vereinbarkeit von Familie und Beruf. Ergebnisse des Mikrozensus 2011", Wirtschaft und Statistik, Dezember, pp. 1079-1099, 1093. https://www.destatis.de/DE/ Publikationen/WirtschaftStatistik/WirtschaftStatistik_Aufsatz. html;jsessionid=827D11D9E80004BD2718C712E2A9972F.cae1 [consultado el 12/7/2013].

4 La esperanza de vida al nacer de los hombres ha aumentado una vez y media más que la de las mujeres ( 0.35 al lugar de 0.23 años) durante los últimos 20 años debido a la disminución de las enfermedades respiratorias, Weiland, pp. C.875y ss., 878.

5 Gracias a las manipulaciones de la industria del tabaco.

6 Rattay, P.; Butschalowsky, H. et al. (2013), Inanspruchnahme der ambulanten und stationären medizinischen Versorgung in Deutschland. Ergebnisse der Studie zur Gesundheit Erwachsener in Deutschland (DEGS1), 56, Bundesgesundheitsblatt, pp. $832-844,835$

7 El mayor consumo de bebidas alcohólicas entre los hombres podría ser el equivalente funcional del consumo de medicamentos psicotrópicos s entre las mujeres.
Dinges, Martin (2011), "Medizin- und gesundheitsgeschichtliche Paradigmen zur geschlechterspezifischen Ungleichheit in der Zeit seit ca. 1750: Von kontrastiv konzipierter Ungleichheit zu intersektional bestimmten Gesundheitsstilen?", Österreichische Zeitschrift für Geschichtswissenschaften, 22 (2), pp. 8-49.

Dinges, Martin (2011a), "Die Gesundheit von Jungen und männlichen Jugendlichen in historischer Perspektive (1780-2010)", Medizin, Gesellschaft und Geschichte, 29, pp. 97-121.

Dinges, Martin (2012), "Rauchen: gesundheitsgefährdend - und typisch „männlich”? Zum historischen Wandel geschlechtsspezifischer Zuschreibungen". En: Baader, Meike S.; Bilstein, Johannes; Tholen, Toni (eds.), Erziehung, Bildung und Geschlecht. Männlichkeiten im Fokus der Gender-Studies, Wiesbaden, Springer VS, pp. 129-145.

Dinges, Martin (2013), "Wandel der Herausforderungen an Männer und Männlichkeit in Deutschland seit 1930". En: Weissbach, Lothar; Stiehler, Matthias (eds.), Männergesundheitsbericht 2013. Im Fokus: Psychische Gesundheit, Bern, Huber, pp. 31-62.

Hoffmann, Annicka (2013), Arzneimittelkonsum und Geschlecht: Eine historische Analyse zum 19. und 20. Jahrhundert, Stuttgart, Steiner. 
Hoffmann, Susanne (2007), "Erwerbsarbeit - Risiko und Ressource für die Gesundheit von Männern: Sechs Autobiographien aus dem 20. Jahrhundert". En Dinges, Martin (ed.), Männlichkeit und Gesundheit im historischen Wandel ca. 1800 - ca. 2000. Stuttgart, Steiner, pp. 241-256.

Hoffmann, Susanne (2010), Gesunder Alltag im 20. Jahrhundert? Geschlechterspezifische Diskurse und gesundheitsrelevante Verhaltensstile in deutschsprachigen Ländern, Stuttgart, Steiner.

Hollstein, Walter (2002), "Der Mann als Täter und Opfer. Die Erkenntnisleistung der Männerforschung für den Kontext von Gesundheit und Krankheit". En: Hurrelmann, Klaus; Kolip, Petra (eds.), Geschlecht, Gesundheit und Krankheit. Männer und Frauen im Vergleich, Bern, Huber, pp. 53-66.

Lengwiler, Martin (2007), “Männliches Risikoverhalten und sozialstaatliche Risikoprofile: Berufskrankheiten des Bergbaus in der schweizerischen Unfallversicherung (1930-1970)". En Dinges, Martin (ed.) (2007), Männlichkeit und Gesundheit im historischen Wandel ca. 1800 - ca. 2000. Stuttgart, Steiner, pp. 257-274.

Meuser, Michael (2007), Der "kranke Mann"- wissenssoziologische Anmerkungen zur Pathologisierung des Mannes in der Männergesundheitsforschung. En Dinges, Martin (ed.), Männlichkeit und Gesundheit im historischen Wandel ca. 1800 - ca. 2000. Stuttgart, Steiner. pp. 73-86.

Resch, Marianne (2002), „Der Einfluss von Familien- und Erwerbsarbeit auf die Gesundheit“. En: Hurrelmann, Klaus; Kolip, Pet- ra (eds.), Geschlecht, Gesundheit und Krankheit. Männer und Frauen im Vergleich, Bern, Huber, pp. pp. 403-418.

Schweig, Nicole (2007), "Familienstand und gesundheitsförderliches Verhalten von Männern in Briefen (1840-1950)". En Dinges, Martin (ed.), Männlichkeit und Gesundheit im historischen Wandel ca. 1800 - ca. 2000. Stuttgart, Steiner, pp. 211-226.

Schweig, Nicole (2008), Gesundheitsverhalten von Männern. Gesundheit und Krankheit in Briefen, 1800 - 1950, Stuttgart, Steiner.

Siegrist, Johannes (2010), "Arbeit, Arbeitslosigkeit und Gesundheit". En: Stiehler, Matthias, Bardehle, Doris (eds.), Erster deutscher Männergesundheitsbericht, München, Zuckerschwerdt, pp. 72-86.

Weigl, Andreas (2007), "Der gender gap- ein Industrialisierungsphänomen? Komparatistische Anmerkungen zu einer schwedischen Fallstudie". En Dinges, Martin (ed.), Männlichkeit und Gesundheit im historischen Wandel ca. 1800 - ca. 2000. Stuttgart, Steiner, pp. 23-40.

Weigl, Andreas (2011), “Arbeit, Lebenserwartung, Geschlecht: Wien 1900-1950", Österreichische Zeitschrift für Geschichtswissenschaften, 22 (2), pp. 112-139.

Weiland, Stephan K. et al. (2006), "Zunahme der Lebenserwartung. Größenordnung, Determinanten und Perspektiven", Deutsches Ärzteblatt 103, pp. C874-C879. 